Städteordnung von 1808 vom Reichsfreiherrn Heinrich Friedrich Karl vom und zum Stein die Selbstverwaltung „erfunden“.

\section{Entwicklung in Deutschland}

Der theoretische Ansatz lag in der Partizipation: die Mitwirkung von Staatsbürgern bei der Erfüllung staatlicher Aufgaben. Es ging natürlich noch nicht um Zahnärzte, sondern Steins Konzept sah die „Reformierung des Staates durch Beteiligung der Nation (Gesellschaft) an der Erfüllung öffentlicher Angelegenheiten“ vor, wie es bei Martin Will in „Selbstverwaltung der Wirtschaft“ heißt. Hiermit wollte Stein die Bürger zu Gemeingeist und Bürgersinn lenken, zur Identifikation mit der Gemeinschaft. Auch wenn Stein den Begriff „Selbstverwaltung“ noch nicht benutzte, so findet sich hier das Element der Partizipation der Betroffenen am Verwaltungsgeschehen.

Es ist etwas irritierend, dass ein weiterer Stein, nämlich Lorenz von Stein, Staatsrechtler, Soziologe und Nationalökonom, in der Mitte des 19. Jahrhunderts sich mit dem Begriff „Selbstverwaltung “ ausführlich befasste und ein weiteres Element neben der Partizipation in den Begriff implementierte: die Freiheit. „Die Selbstverwaltung ist daher die erste, ich möchte sagen die natürliche Grundform der freien Verwaltung überhaupt; solange sie noch anerkannt ist und wirkt, gibt es eine freie Verwaltung im Staate; wo sie verschwindet, verschwindet überhaupt das Element der Freiheit in der Verwaltung; wo sie blüht, ist die letztere gesichert.“

Für Lorenz von Stein ist Selbstverwaltung das zentrale Prinzip, um einen Ausgleich politischer Gewichte der sich rasch verändernden Gesellschaft herbeizuführen. Partizipation und Selbstbestimmung sind für ihn Kern der Selbstverwaltung, allerdings nur, wenn die Betroffenen teilnehmen können und wollen.

Ein weiteres tragendes Element in der Selbstverwaltung ist das Prinzip der Subsidiarität. Sowohl der Liberalismus als auch die katholische Soziallehre stellten im 19. Jahrhundert fest, dass der Staat nicht alle Aufgaben an sich ziehen solle, solange diese von Einzelnen genauso gut oder sogar besser erledigt werden können.

\section{Umkehrung des Prinzips}

Die geschichtlichen Ereignisse ließen eine dauerhafte Etablierung des Prinzips Selbstverwaltung mit seinen theoretischen Grundlagen Partizipation, Freiheit und Subsidiarität nicht zu. Zwar wurde der Begriff Selbstverwaltung weiterverwendet, aber bereits die Politik der Notverordnungen in der Weimarer Republik und erst recht das Führerprinzip im Dritten Reich waren mit den theoretischen Grundlagen nicht vereinbar. Die bis dahin bestehenden Organisationen der Selbstverwaltung verloren ihre Freiheitsgrade, ihre Partizipation bestand darin, die Kosten der Verwaltungsorganisation selbst zu tragen, „Subsidiarität“ hieß, die obrigkeitlichen Befehle unmittelbar im „Sinne des Führerbefehls“ selbstständig umzusetzen.

Anhand dieser Entwicklung wird klar, dass eine echte Selbstverwaltung ohne die Grundlagen Partizipation, Freiheit und Subsidiarität nicht erreichbar ist. Demokratische Bedingungen in der Selbstverwaltung (beispielsweise Wahlfreiheit und Wählbarkeit) sind heutzutage eine Conditio sine qua non, aber kein eigenständiges Merkmal. Wesentlicher ist es, sich freiwillig zusammenschließen zu können (Freiheit) und zu dürfen (Partizipation) und über alle Aufgaben entscheiden zu können, die die kleinere Einheit genauso gut oder besser erledigen kann (Subsidiarität).

\section{Falsch verstandene Rechtsaufsicht}

Kameralistik ist ein anderes Wort für "staatliche Verwaltungswissenschaft". Wer in der öffentlichen Verwaltung tätig ist, sollte also einige Grundbegriffe von der staatlichen Verwaltung (Rechnungsführung, Finanz-, Wirtschafts-, Verwaltungslehre, Rechts- und Polizeiwissenschaft) und Volkswirtschaftspolitik kennen.

Umso erstaunlicher ist es, in jüngster Zeit seitens einiger Aufsichtsbehörden zu hören, dass die von den Kassenzahnärztlichen Vereinigungen verwalteten Finanzmittel "Versichertengelder" seien. Es scheint inzwischen in Vergessenheit geraten zu sein, dass es sich um "Zahnärztegelder" handelt. "Versichertengelder" sind das Finanzvolumen der Krankenkassen, mit dem die Krankenkassen die Behandlungen der Zahnärzte für ihre Versicherten bezahlen. Die Finanzmittel der KZVen bestehen ausschließlich aus dem erarbeiteten Honorar der Zahnärzte.

\section{Grundbedingungen heute nicht erfüllt}

Im Gesundheitswesen finden wir den Begriff Selbstverwaltung oft wieder. Krankenkassen, Ärztekammern, KZVen, Gemeinsamer Bundesausschuss und andere mehr werden als Selbstverwaltungen bezeichnet. Ob sie die drei Grundbedingungen erfüllen, muss mit Recht bezweifelt werden.

Die Mitgliedschaft in einer Krankenkasse ist nicht freiwillig, auf ihr Verwaltungshandeln hat das Mitglied keinen Einfluss, ihr Organisationsprinzip ist bis in das kleinste Detail durch die Sozialgesetzbücher vorgeschrieben. Die Krankenkasse kann nicht über ihre Mittelherkunft entscheiden, ihr Aufgabenspektrum wird staatlich festgelegt, es werden ihr willkürlich sachfremde Aufgaben zugeteilt.

Bei den (zahn)ärztlichen Selbstverwaltungsorganen sieht es ähnlich aus: Die Mitgliedschaft ist nicht freiwillig, das Organisationsprinzip und das Aufgabenspektrum sind bis in die Detailtiefe festgelegt. Die Mittelverwendung ergibt sich aus dem Sozialgesetzbuch 5 (SGB V) oder aus den Kammergesetzen. Disziplinierungspflichten gegenüber den Mitgliedern sind gesetzlich vorgegeben, es gilt das Verwaltungsrecht genauso wie bei der unmittelbaren Staatsverwaltung.

Schon immer unterlagen die staatlich eingerichteten Selbstverwaltungen der Rechtsaufsicht durch den Staat. Die Rechtsaufsicht hatte zu prüfen, ob das Verwaltungshandeln der Selbstverwaltung die gesetzlichen Rahmenbedingungen einhielt. Solange die Rahmenbedingungen tatsächlich nur den „großen Rahmen“ vorgeben, lässt sich dies mit den drei Grundbedingungen durchaus in Einklang bringen. Inzwischen kann man aber nicht mehr von "Rahmenbedingungen“ sprechen, da das Verwaltungshandeln bis in das kleinste Detail vorgegeben ist. Von einer Autonomie kann nicht mehr gesprochen werden.

\section{Unmittelbare Staatsverwaltung}

Aus staatstheoretischen Gründen spricht alles für eine echte Selbstverwaltung. Zumindest im Gesundheitswesen gibt es sie nicht. Es ist nicht abwegig zu behaupten, dass wir Zahnärzte uns eigentlich in einem System unmittelbarer Staatsverwaltung befinden, mit dem einzigen Unterschied: Wir haben sie selbst zu bezahlen. Und so sagen sich vielleicht viele Kollegen, König Friedrich August III. von Sachsen zitierend: „Nu da machd doch eiern Drägg alleene.“

Bertram Steiner 